\title{
Autonomic regulation of imbalance-induced myocardial fibrosis and its mechanism in rats with cirrhosis
}

\author{
SHANSHAN YU*, LEI SUN*, HUA WANG, JUE JIANG and QI ZHOU \\ Department of Ultrasound, The Second Affiliated Hospital of Xi'an Jiaotong University, Xi'an, Shaanxi 710004, P.R. China
}

Received December 11, 2019; Accepted June 10, 2021

DOI: $10.3892 / \mathrm{etm} .2021 .10472$

\begin{abstract}
The aim of the present study was to investigate the changes in cardiac function and myocardial damage in rats with cirrhosis. In addition, a secondary aim was to explore any potential changes in the expression levels of $\beta 1$-adrenergic $(\beta 1)$ and muscarinic acetylcholine (M2) receptors . A cirrhotic cardiomyopathy (CCM) rat model was established by CCL4-oil solution for subcutaneous injection into the neck. Pathological changes in the liver and myocardial tissues were detecting by H\&E staining and Masson trichrome staining. Furthermore, changes in the levels of myocardial enzymes lactate dehydrogenase ( $\mathrm{LDH})$, creatine kinase isoenzyme (CK-MB) and troponin in serum were measured by ELISA. The myocardial samples were homogenized and centrifuged. Subsequently, the supernatant was collected for detecting the expression of interleukins in myocardial tissue. Changes in the levels of inflammatory factors, IL-1, IL-2 and IL- 6 both in the serum and myocardial tissue were determined by ELISA. Changes in echocardiographic measurements were evaluated using high-frequency ultrasound and the expression levels of $\beta 1$ and M2 receptors in myocardial tissues were determined by western blotting. The normal lobular structure in liver tissues was found to be disappeared 8 weeks after modeling, which was replaced by pseudolobules in the rats in the CCM group. In addition, the myocardial cells were observed to be swollen and disorderly arranged. Compared with those in the control group, the left ventricular end-systolic and end-diastolic dimensions, interventricular septal dimension and LAD in rats in the CCM8 group were found to be significantly increased. The levels of myocardial enzymes LDH, CK-MB and cardiac troponin in the serum were also revealed to be significantly increased in the CCM8 group. Additionally, the levels of IL-1
\end{abstract}

Correspondence to: Professor Qi Zhou, Department of Ultrasound, The Second Affiliated Hospital of Xi'an Jiaotong University, 157 Xiwu Road, Xi'an, Shaanxi 710004, P.R. China

E-mail: docqizhou@163.com

${ }^{*}$ Contributed equally

Key words: cirrhotic cardiomyopathy, rat, high frequency ultrasound, pathogenesis and IL- 6 in both serum and myocardial tissues were significantly increased in rats in the CCM8 group. However, the levels of IL-2 in both serum and myocardial tissues were decreased, which were observed alongside reductions in myocardial $\beta 1$ and $\mathrm{M} 2$ receptor protein expression in the myocardial tissues. Taken together, these results indicate that inflammatory factors may be involved in mediating damage to the myocardium in rats with cirrhosis. During cirrhosis-induced cardiac dysfunction, there may exist a mechanism for downregulation of autonomic nerve system.

\section{Introduction}

Cirrhosis is a liver disease that is characterized by widespread necrosis, inflammation and fibrosis in the liver tissues (1). During cirrhosis, nodular regeneration of residual hepatocytes also occurs, which is caused by the chronic action of various factors including inflammation, activation of hepatic stellate cells with ensuing fibrogenesis, angiogenesis and parenchymal extinction lesions caused by vascular occlusion (2). In addition to liver damage, cirrhosis can also result in a series of other systemic impairments. In 1953, Kowalski and Abelmann first documented abnormal cardiac functions in patients with liver cirrhosis (3). Since then, a series of animal models and clinical studies have confirmed further that myocardial contractility and stimulation in patients with cirrhosis are weakened, where in severe cases heart failure can occur (4-6). This condition is known as cirrhotic cardiomyopathy (CCM) (4-6). Previous studies have shown that echocardiography (ECG) can be used to evaluate left ventricular diastolic functions in patients with cirrhosis $(7,8)$. In addition, other studies successfully used ultrasound speckle tracking echocardiography to detect reduced systolic functions in patients with cirrhosis during the early stages of the disease (9). Cirrhosis can hinder contractions and diastolic functions of the heart and can cause heart failure in severe cases (9). Although myocardial remodeling has been proposed to be a cause of this phenomena in cardiac functions during cirrhosis (9), it remains unclear how reconstruction of the myocardium occur in such cases and how these pathological changes affect cardiac function.

The specific pathogenesis of CCM remain poorly understood. Recent studies have suggested that abnormalities in the $\beta$-adrenergic receptor ( $\beta$-AR) signaling pathway are associated with the development of CCM $(10,11)$. $\beta$-AR is the major class of receptor that regulates cardiac functions by exerting 
positive chronotropic, inotropic and dromotropic actions (10). Downregulating the expression of $\beta$-AR at the onset of CCM has been reported to reduce myocardial contractility (12). However, the effects of $\beta$-AR and other inflammatory factors including tumour necrosis factor (TNF)- $\alpha$, interleukin (IL)- $1 \beta$, IL-6 and IL-18 (13), which are mediated by the $\beta$-AR signaling pathway during myocardial injury, on cardiac physiology during CCM remain poorly understood.

The heart is regulated by the autonomic nervous system, where a dynamic balance is maintained by the homeostasis of sympathetic and parasympathetic stimulation (14). However, no reports of changes in parasympathetic myocardial stimulation during CCM have been previously reported. Therefore, the present study established a carbon tetrachloride (CCL4)-induced CCM rat model for assessing changes in the expression profiles of $\beta$-AR and muscarinic acetylcholine (M2) receptors along with the activation of their corresponding signal transduction pathways. The aim is to explore the underlying mechanism of CCM and to provide a theoretical and practical basis for developing clinical prevention and treatment strategies for CCM.

\section{Materials and methods}

Animals, modeling and grouping. In total, 40 male Sprague-Dawley (SD) rats aged 6-7 weeks weighing 180 $\pm 20 \mathrm{~g}$ were provided by the Experimental Animal Center of Xi'an Jiaotong University Medical College and randomly divided into the following three groups: i) Control group ( $\mathrm{CON} ; \mathrm{n}=10)$; ii) 4-week CCM group (CCM4; n=15); and iii) 8-week CCM group (CCM8; n=15). The rats in the present study were maintained on a standard rat chow diet with free access to drinking water. Four or five animals were housed per polycarbonate cage under controlled conditions $\left(22 \pm 2^{\circ} \mathrm{C}, 50-60 \%\right.$ relative humidity and 12-h light/dark cycles).

For treatment, CCL4 (99.5\% analytical pure supplied by Shanghai Siyan Biotechnology Co., Ltd.) and soybean oil were formulated into 40 and $60 \%$ CCL4-oil solution for subcutaneous injection into the neck of each rat. In the CCM4 group, rats received an intraperitoneal injection of $40 \%$ CCL4-oil solution at weeks 1 and 2, twice per week (Monday and Thursday), with the first dose at $5 \mathrm{ml} / \mathrm{kg}$ and the remaining (including the second, the third and the fourth) dose at $3 \mathrm{ml} / \mathrm{kg}$, and fed with $10 \%$ ethanol in drinking water (every other day). On weeks 3 and 4, each rat received a subcutaneous injection of 60\% CCL4-oil solution twice/week (Monday and Thursday), with the dose as $3 \mathrm{ml} / \mathrm{kg}$ and fed with $20 \%$ ethanol in drinking water (every other day). The rats were then subcutaneously injected with the same amount of $0.9 \%$ normal saline from weeks 5 to 8, twice per week (Monday and Thursday) and feed with common feed and tap water.

For rats in the CCM8 group, they received an intraperitoneal injection of 40\% CCL4-oil solution on weeks 1 and 2 twice per week (Monday and Thursday), with the first dose as $5 \mathrm{ml} / \mathrm{kg}$ and the remaining dose at $3 \mathrm{ml} / \mathrm{kg}$. During feeding, $10 \%$ ethanol solution was added to drinking water every other day. On weeks 3 to 8 , rats received a subcutaneous injection of $60 \%$ CCL4-oil solution, twice per week (Monday and Thursday), with the dose set to $3 \mathrm{ml} / \mathrm{kg}$ and $20 \%$ ethanol solution was added to drinking water every other day.
The rats in CON group were given normal drinking water and granulated feed throughout the 8 weeks. The CON group was intraperitoneally injected with the same amount of $0.9 \%$ normal saline twice per week (Monday and Thursday) and then subcutaneously injected with the same amount of $0.9 \%$ normal saline from weeks 3 to 8 (twice per week, on Monday and Thursday).

During this experimental period, the general condition of the rats, including coat color, activity, appetite and death, were observed every day, together with the final body weight of each rat. When the rats developed cachexia or extreme weakness, euthanasia would be immediately performed. The standard used to assess the successful establishment of cirrhosis model in the rats were as follows: i) Biochemical indices, including increased levels of alanine aminotransferase (ALT) and aspartate aminotransferase (AST), reduced levels of total protein (TP) compared with those in the control group; ii) Imaging indices, which were assessed using ultrasonography, whereby compared with those in the control group, liver edges are round and blunt (sharp edge of the liver in the control group rats) but the echo light spot of the parenchyma are increased (uniform light spot in the control group); and iii) Histopathological changes in the liver as assessed by H\&E staining, whereby hepatic lobules were destroyed, can be observed with fatty deposits and fibrous hyperplasia wrapping around the liver cell mass to form a typical pseudolobule (in the control group, the structure of liver lobule should be normal, where hepatocytes around the central portal vein are arranged radially) (15). There were no deaths in the CON group. A total of 3 rats died in the CCM4 group and 5 rats died in the CCM8 group. Specifically, two rats reached the humane endpoints by showing clear anorexia and depression, weight loss and cachexia. In total, three rats had to be euthanized as they developed severe weakness and were unable to stand. One rat developed severe infection and ulceration at the injection site, which was euthanized immediately. Two rats were found dead when fed early morning. It was speculated that the death may have been caused by cachexia and extreme weakness. All animal procedures conformed to the guidelines for the Care and Use of Laboratory Animals published by the National Institutes of Health (NIH publication 85-23, revised in 1996) (16) and the approved regulations set by the Laboratory Animal Care Committee at Xi'an Jiaotong University (Xi'an, China).

ECG measurements. The instrument used for ECG measurement was Hitachi HI VISION Preirus color ultrasound diagnostic instrument (Hitachi High-Technologies Corporation) equipped with a EUP-L74M linear array probe (probe frequency, 5.0-13.0 MHz; Hitachi High-Technologies Corporation). All rats were weighed prior to ECG analysis and were anesthetized with an intraperitoneal injection of $2 \%$ pentobarbital sodium $(30 \mathrm{mg} / \mathrm{kg})$. ECG was performed on anesthetized rats fixed in a supine position. The parasternal long and short axis sections of the left ventricle were taken for 2D ultrasound measurements using an M-shaped curve. Parameters measured included the following: i) Left ventricular posterior wall dimension (LVPW); ii) interventricular septal dimension (IVS); iii) left ventricular end-diastolic dimension (LVEDD); iv) left ventricular end-systolic dimension (LVESD); v) left atrial diameter (LAD); vi) left ventricular ejection 


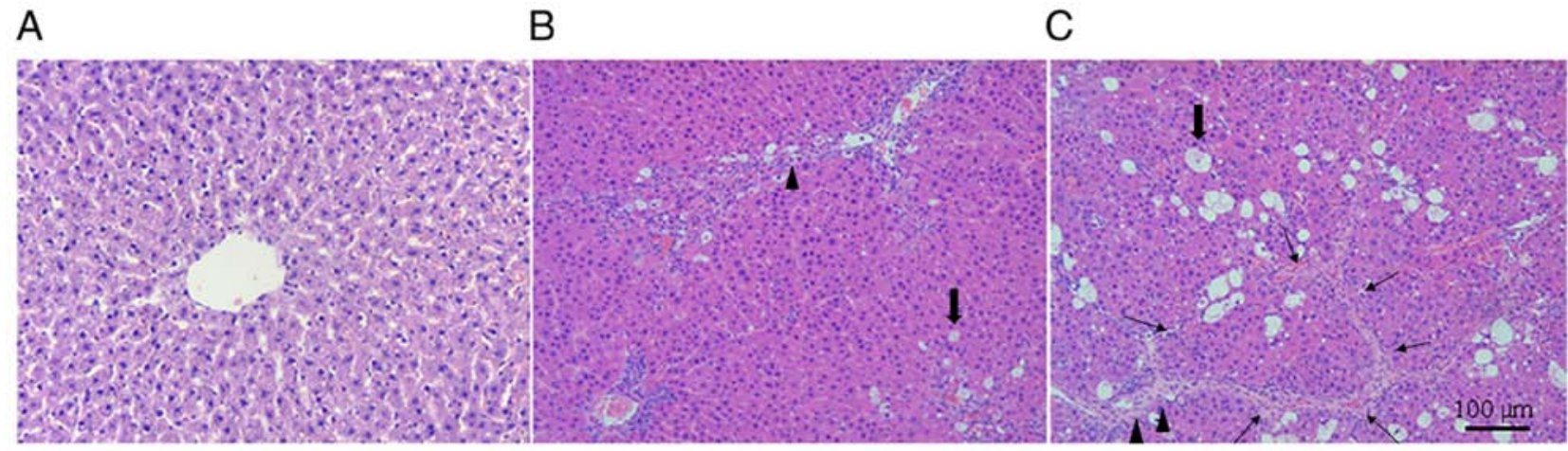

Figure 1. H\&E staining of liver tissues in the three treatment groups. (A) Control group, (B) 4-week cirrhotic cardiomyopathy group and (C) 8-week cirrhotic cardiomyopathy group. Magnification, x100. Thick arrow, steatosis; arrow head, fiber proliferation; thin arrow, formation of pseudolobule.

fraction (LVEF); vii) left ventricular short axis shortening rate (FS); and viii) cardiac output (CO). Each index was measured over three consecutive cardiac cycles and averaged.

Sampling of serum and tissue specimens. After ECG measurements, the rats were euthanized by $\mathrm{CO}_{2}$ inhalation (30\% volume per minute displacement) followed by cervical dislocation. Upon completion of the procedure, death was confirmed by observation of cardiac and respiratory arrest or fixed and dilated pupils. All the experimental operations were in accordance with the requirements of the Experimental Animal Ethics Committee, School of Medicine, Xi'an Jiaotong University. All rats were cut open near the abdominal cavity and $\sim 5 \mathrm{ml}$ blood was taken for sampling from the abdominal aorta. The blood samples were then kept at room temperature for $30 \mathrm{~min}$, followed by centrifugation at $1,509 \mathrm{x} \mathrm{g}$ at $4^{\circ} \mathrm{C}$ for $15 \mathrm{~min}$ before the serum was collected and stored at $-80^{\circ} \mathrm{C}$. In total, $500 \mu 1$ serum from each sample was taken out and thawed. The levels of myocardial enzymes, specifically lactate dehydrogenase (LDH), creatine kinase isoenzyme (CK-MB) and cardiac troponin $\mathrm{T}$ (cTnT), along with liver function markers ALT, AST and TP, were determined using a Beckman Coulter LH750 automatic biochemical analyzer (Beckman Coulter, Inc.) at the Department of Clinical Laboratory, School of Medicine, The Second Affiliated Hospital of Xi'an Jiaotong University. An incision was then made in the chest cavity for collecting the liver and heart. After the blood was rinsed using saline and the tissues were dried using filter paper, their corresponding wet weights were measured. In each rat, exactly the same region of the right liver lobe and myocardial apex were collected, fixed in $4 \%$ paraformaldehyde at $4^{\circ} \mathrm{C}$ for $24 \mathrm{~h}$ and embedded in paraffin for $\mathrm{H} \& \mathrm{E}$ and Masson trichrome staining. The rest of the myocardial and liver samples were placed in separately cryotubes and rapidly frozen in liquid nitrogen, which were stored at $-80^{\circ} \mathrm{C}$ for future protein detection measurements. Before detecting the expression of interleukins in myocardial tissue, the myocardial samples of rats were taken and kept at $\sim 2-8^{\circ} \mathrm{C}$ with a certain amount of PBS (pH 7.4). The samples were homogenized by homogenizer and centrifuged at $1,509 \mathrm{x}$ g for about $20 \mathrm{~min}$ at $4^{\circ} \mathrm{C}$. The supernatant was collected for detecting the expression of interleukins in myocardial tissue. The levels of IL-1, IL-2 and IL-6 in the serum and myocardial tissue were measured with ELISA kits (Wuhan USCN Business Co., Ltd.) in accordance with the manufacturer's protocol. The catalogue numbers of IL-1, IL-2 and IL-3 were SEA563Ra, SEA073Ra and SEA079Ra, respectively.

$H \& E$ staining and Masson trichrome staining. For $\mathrm{H} \& \mathrm{E}$ staining, after the 5- $\mu \mathrm{m}$ thick paraffin slice was dewaxed with xylene and then fully hydrated with ethanol, it was stained with a hematoxylin solution for 3-8 $\mathrm{min}$ at room temperature, followed by rinsing with water, $1 \% \mathrm{HCl}$-alcohol differentiation for 5-30 sec, another water rinsing step, incubation in $0.6 \%$ ammonia water solution for bluing for $1.5 \mathrm{~h}$ and staining with an eosin dye solution for 1-3 min at room temperature. The sections were then dehydrated and hyalinized in xylene before finally being sealed in neutral gum. All the slides were examined under a light microscope and images were captured with a Nikon microscope (Y-THS; Nikon Corporation) (magnification, x100).

For Masson trichrome staining, after the $5-\mu \mathrm{m}$ thick paraffin slice was dewaxed with xylene and then fully hydrated with ethanol, it was placed in a dichromate-acetic acid solution for $40 \mathrm{~min}$ at room temperature, followed by a water rinsing, hematoxylin staining for $10 \mathrm{~min}$ at room temperature, $2 \%$ $\mathrm{HCl}$-alcohol differentiation, 15 -min staining in $1 \%$ ponceau acid fuchsin at room temperature, 5-min treatment in $1 \%$ phosphomolybdic acid, 8 -min staining with $2 \%$ bright green solution at room temperature and 2 to 3 -time color separation in $1 \%$ acetic acid. The samples were then dehydrated/hyalinized before being finally sealed using in neutral gum. All the slides were examined under a light microscope and images were captured with a Nikon microscope (Y-THS; Nikon Corporation) (magnification, $\mathrm{x} 400$ ).

Western blotting. The myocardial tissue was cryopreserved in liquid nitrogen. Then, RIPA buffer (cat. no. R0010; Beijing Solarbio Science \& Technology Co., Ltd.) and a protease inhibitor [Roche Diagnostics (Shanghai) Co., Ltd.] were used for total protein extraction. Protein concentrations were quantified by BCA assay (cat. no. PA115-01, Tiangen Biotech Co, Ltd.) and diluted to a final concentration of $5 \mu \mathrm{g} / \mu \mathrm{l}$. The subsequent $10 \%$ SDS-PAGE was performed as follows: i) $50 \mu \mathrm{g}$ protein per lane; ii) $90 \mathrm{~min} 280 \mathrm{~mA}$ constant-current electrophoresis; iii) $1 \mathrm{~h}$ blocking in 5\% skim milk at room temperature; iv) overnight incubation in primary antibody at $4^{\circ} \mathrm{C}$; and v) $1 \mathrm{~h}$ incubation with secondary antibodies at $37^{\circ} \mathrm{C}$. Primary 
Table I. Comparison of liver function parameters among the three groups.

\begin{tabular}{lccc}
\hline Parameter & CON & CCM4 & CCM8 \\
\hline Aspartate aminotransferase & $72.3 \pm 7.5$ & $102.8 \pm 10.8^{\mathrm{b}}$ & $198.7 \pm 12.5^{\mathrm{b}}$ \\
Alanine aminotransferase & $65.1 \pm 7.0$ & $89.4 \pm 9.6^{\mathrm{a}}$ & $132.0 \pm 11.9^{\mathrm{b}}$ \\
Total protein & $54.7 \pm 6.2$ & $46.2 \pm 4.3^{\mathrm{a}}$ & $37.2 \pm 4.0^{\mathrm{b}}$
\end{tabular}

${ }^{\mathrm{a}} \mathrm{P}<0.05$ and ${ }^{\mathrm{b}} \mathrm{P}<0.01$ vs. CON. CON, control; CCM4, 4-week cirrhotic cardiomyopathy; CCM8, 8-week cirrhotic cardiomyopathy.

A

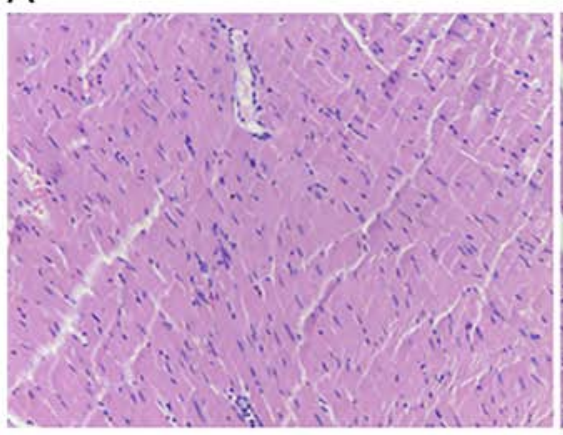

B

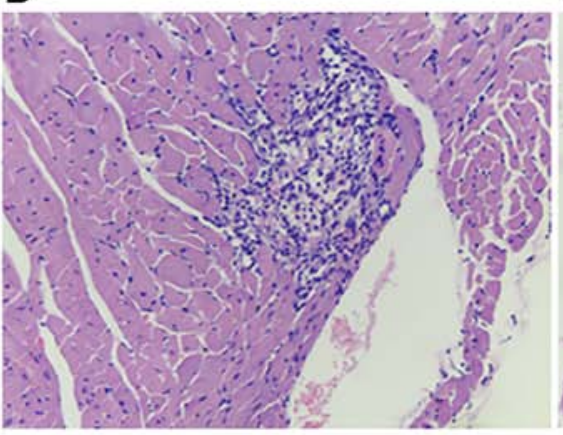

C

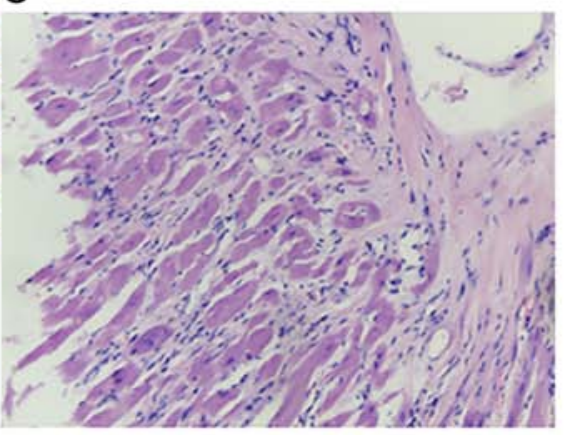

D

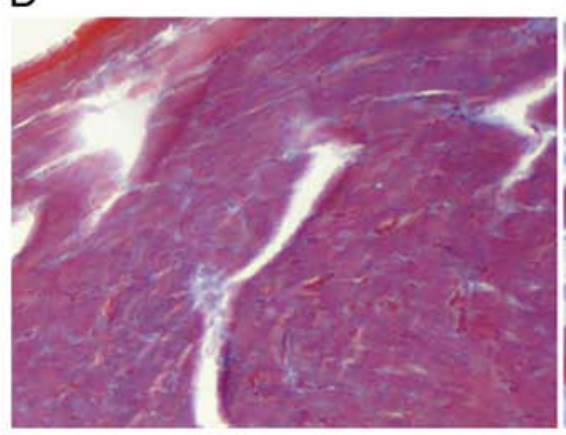

E

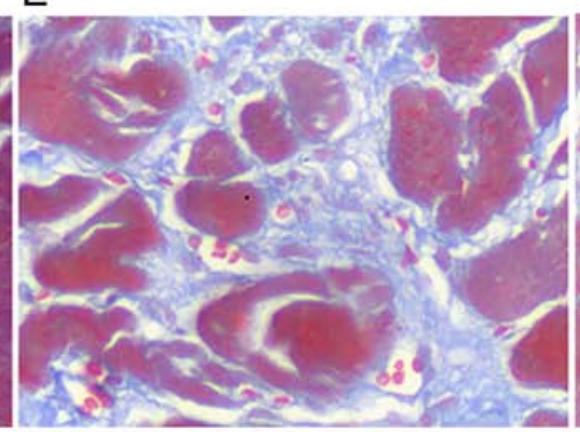

$\mathrm{F}$

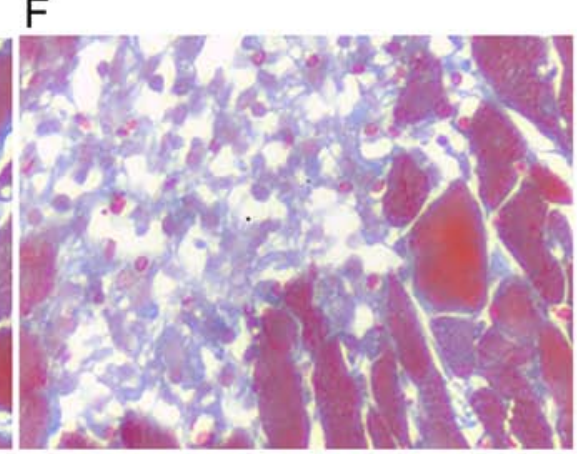

Figure 2. Cardiac histopathology in the three treatment groups. (A-C) Representative H\&E staining images. (A) Control group, (B) 4-week cirrhotic cardiomyopathy group and (C) 8-week cirrhotic cardiomyopathy group. Magnification, x100. (D-F) Representative Masson trichrome staining images. (D) Control group, (E) 4-week cirrhotic cardiomyopathy group and (F) 8-week cirrhotic cardiomyopathy group. Magnification, x400.

antibodies used include anti-GAPDH (cat. no. sc-365062, 1:10,000; Santa Cruz Biotechnology, Inc.), anti-mAChR M2 (cat.no. sc-33712, 1:1,000; Santa Cruz Biotechnology, Inc.) and anti- $\beta 1$ adrenergic receptor (cat. no. sc-81577, 1:1,000; Santa Cruz Biotechnology, Inc.). The secondary antibodies used were goat anti-mouse IgG1-HRP (cat. no. SC-2060, 1:10,000; Santa Cruz Biotechnology, Inc.) and goat anti-mouse IgG2a-HRP (cat. no. SC-2970, 1:10,000; Santa Cruz Biotechnology, Inc.). The gel strips were then photographed by luminescent imaging (Tanon 6200 Luminescent Imaging Workstation; Tanon Science and Technology Co., Ltd.). Quantification was performed using Image J v.1.8.0 (National Institutes of Health). GAPDH was used as the loading control.

Statistical analysis. The data were expressed as the mean \pm SD. SPSS 18.0 (SPSS, Inc.) statistical analysis software was used to perform one-way ANOVA followed by the LSD post hoc test. $\mathrm{P}<0.05$ was considered to indicate a statistically significant difference.

\section{Results}

Changes in liver structure and function in the CCM rat models. H\&E staining under light microscopy showed that after CCM induction, the hepatocytes present in liver tissues had varying degrees of hepatic lobules destroyed and steatosis, where the fibers proliferated significantly and formed a typical pseudolobule around the hepatocyte mass (Fig. 1). In addition, the extent of this appeared to be more severe in liver tissues from rats in the CCM8 group (Fig. 1). This suggested that the CCM model was successfully established.

Serum levels of alanine aminotransferase (ALT) and aspartate aminotransferase (AST) are indicators of hepatocyte injury (17). ALT and AST combined with liver histopathology were used in the present study to confirm whether the model group was successfully modeled and the degree of hepatocyte injury. Compared with those in the CON group, the concentrations of ALT and AST were significantly increased, whilst those of TP were significantly decreased, in both the 
CCM4 and the CCM8 groups. ALT and AST levels were also significantly increased in the CCM8 group compared with those in the CCM4 group (Table I).

Changes in the myocardial structure and function after CCM induction. Morphological changes in the myocardial tissue of rats after CCM induction was assessed using H\&E staining. Cardiomyocytes from rats in the CON group were arranged tightly in a regular manner, with their full structure intact and clear cell boundaries clearly visible. In the CCM model of rats, the myocardial structure was more disordered with nuclei accumulation (Fig. 2B and C). Furthermore, the myocardial fibers were either swollen, dissolved or broken (Fig. 2B and C). Masson trichrome staining is frequently used for detecting tissue collagens, which can distinguish among muscle fibers, collagen fibers and nuclei (18). Masson trichrome staining results revealed that the cardiomyocytes of the rats in the CON group were orderly arranged with intact muscle bundles. The myocardial tissue had a small quantity of collagen fibers, which stain blue and were distributed evenly among the red cardiomyocytes. After CCM induction, myocardial tissue damage appeared to have occurred in rats in both CCM4 and CCM8 groups (Fig. 2). Features observed include basic tissue destruction, large-areas fibrotic tissues displacing the healthy myocardial tissue, excessive myocardial interstitial collagen deposition, a large quantity of fibrous, meshed connective tissues and damaged myocardial muscle bundles, which were either separated, enveloped or fused (Fig. 2).

Compared with those in the CON group, the body weights (BW) and wet heart weights (HW) of the rats in the CCM groups were significantly lower, though the magnitude of decrease was greater in those in the CCM8 group. This may be due to reductions in appetite and water intake in rats in the CCM groups. However, the left ventricular weight (LVW), $\mathrm{HW} / \mathrm{BW}$ and LVW/HW in rats in the CCM group were significantly higher compared with those in the CON group (Table II). This suggest that the heart, especially the left ventricle, had undergone myocardial tissue remodeling.

Compared with those in rats in the CON groups, LVESD, LVEDD, IVS and LAD values in the CCM8 group were significantly increased (Table III). Rats in the CCM8 group also exhibited statistically increased CO, decreased VE/VA ratio and prolonged deceleration time (DT; Table III). There were no significant changes in the posterior wall thickness of the left ventricle and the LVEF values (Table III, Fig. 3).

Changes in levels of myocardial enzymes and inflammatory factors in the serum and myocardial tissues. Compared with those in the CON group, the levels of LDH, CK-MB and cTnT in CCM4 and CCM8 groups were significantly increased (Table IV). In addition, compared with those in the CON group, the levels of pro-inflammatory factors IL-1 and IL-6 in the serum and myocardial tissues were significantly increased in the CCM4 and CCM8 groups (Fig. 4). By contrast, the levels of IL-2 were decreased, but only those in the CCM8 group exhibited statistically significant differences (Fig. 4).

Downregulation of myocardial $\beta 1$ and $M 2$ receptor expression in CCM rats. Semi-quantitative analysis of $\beta 1$ and $\mathrm{M} 2$ receptor protein expression in the myocardial tissues was performed
Table II. Comparison of BW and HW among the three groups.

\begin{tabular}{lrrr}
\hline Parameter & \multicolumn{1}{c}{ CON } & CCM4 & \multicolumn{1}{c}{ CCM8 } \\
\hline BW $(\mathrm{g})$ & $345 \pm 11.4$ & $328.3 \pm 13.0^{\mathrm{a}}$ & $303.0 \pm 9.7^{\mathrm{b}}$ \\
HW $(\mathrm{mg})$ & $987.2 \pm 61.9$ & $967.4 \pm 49.6^{\mathrm{a}}$ & $923.7 \pm 46.3^{\mathrm{a}}$ \\
LVW $(\mathrm{mg})$ & $684.8 \pm 33.7$ & $701.5 \pm 30.1^{\mathrm{a}}$ & $725.6 \pm 15.0^{\mathrm{a}}$ \\
HW/BW & $2.83 \pm 0.11$ & $2.95 \pm 0.16^{\mathrm{a}}$ & $3.04 \pm 0.21^{\mathrm{a}}$ \\
LVW/HW & $0.73 \pm 0.03$ & $0.82 \pm 0.05^{\mathrm{b}}$ & $0.83 \pm 0.04^{\mathrm{b}}$ \\
\hline
\end{tabular}

${ }^{\mathrm{a}} \mathrm{P}<0.05$ and ${ }^{\mathrm{b}} \mathrm{P}<0.01$ vs. $\mathrm{CON}$. BW, body weight; $\mathrm{HW}$, whole heart weight; LVW, left ventricular weight; CON, control; CCM4, 4-week cirrhotic cardiomyopathy; CCM8, 8-week cirrhotic cardiomyopathy.

using western blotting. Compared with that in the CON group, expression of $\beta 1$ and $\mathrm{M} 2$ receptor proteins in the CCM4 and CCM8 groups were significantly downregulated, which was more prominent in the CCM8 group (Fig. 5).

\section{Discussion}

CCM is one of the complications that can occur during cirrhosis (19). Although patients may not exhibit symptoms under normal circumstances, other factors, including stress, infection, transjugular intrahepatic portosystemic shunts (TIPS) or liver transplantation, can result in serious complications (20). Due to CCM being difficult to diagnose early, an increasing number of studies have placed focus on investigating the pathogenesis of left ventricular systolic and diastolic dysfunctions during CCM. The ultimate aim was to devise a method for a more accurate diagnosis and prevention of such complications $(21,22)$. A number of studies $(23,24)$ have reported that compensatory adaptions occur in cardiac function during the early stages of cirrhosis, reported a submaximal increase in cardiac output, heart rate and increase in plasma volume, but the arterial pressure drops and peripheral resistance is reduced. Previous clinical studies have also found that patients with cirrhosis may have systolic and diastolic dysfunctions $(4,25)$. Myocardial remodeling may be the cause of cardiac dysfunction in CCM (20), but the cellular mechanism involved in this remodeling process and how these pathological changes affect cardiac functions remain poorly understood. Since it is difficult to obtain myocardial tissues from patients with cirrhosis, the present study used rats models of CCM to simulate myocardial damage. In the present study, a CCM model was established by CCL 4 treatment, which resulted in ventricular remodeling and fibrosis, in turn leading to left ventricular systolic and diastolic dysfunction. In addition, CCM induced inflammatory reaction in the body, which was characterized by increased levels of proinflammatory factors IL-1 and IL- 6 and decreased levels of the anti-inflammatory factor IL-2. Therefore, it can be speculated that the release of inflammatory mediators serve an important role in myocardial remodeling during CCM (26). Liver cirrhosis can affect a variety of organs and systems in the body, including the cardiovascular and autonomic nervous systems $(27,28)$. Myocardial remodeling and abnormal inflammatory reactions in CCM may be caused by defects in its autonomic nervous regulatory system. 
Table III. High-frequency ultrasound data among the two groups.

\begin{tabular}{|c|c|c|c|c|c|c|c|c|c|c|}
\hline Groups & $\mathrm{n}$ & $\begin{array}{l}\text { LVESD } \\
(\mathrm{mm})\end{array}$ & $\begin{array}{l}\text { LVEDD } \\
(\mathrm{mm})\end{array}$ & $\begin{array}{l}\text { IVS } \\
(\mathrm{mm})\end{array}$ & $\begin{array}{c}\text { LVPW } \\
(\mathrm{mm})\end{array}$ & $\begin{array}{l}\text { LAD } \\
(\mathrm{mm})\end{array}$ & $\begin{array}{l}\text { LVEF } \\
(\%)\end{array}$ & $\mathrm{CO}(\mathrm{l})$ & VE/VA & $\begin{array}{c}\text { DT } \\
\text { (msec) }\end{array}$ \\
\hline $\mathrm{CON}$ & 10 & $3.8 \pm 0.55$ & $6.8 \pm 0.45$ & $1.31 \pm 0.09$ & $1.63 \pm 0.08$ & $4.08 \pm 0.33$ & $77.20 \pm 2.41$ & $0.16 \pm 0.02$ & $1.26 \pm 0.29$ & $18 \pm 2.06$ \\
\hline CCM8 & 10 & $4.4 \pm 0.64^{\mathrm{a}}$ & $7.5 \pm 0.64^{\mathrm{a}}$ & $1.85 \pm 0.25^{\mathrm{a}}$ & $1.84 \pm 0.07$ & $6.67 \pm 0.58^{\mathrm{a}}$ & $75.02 \pm 1.88$ & $0.38 \pm 0.05^{\mathrm{a}}$ & $1.01 \pm 0.16^{\mathrm{a}}$ & $21 \pm 1.23^{\mathrm{a}}$ \\
\hline
\end{tabular}

${ }^{\mathrm{a}} \mathrm{P}<0.05$ vs. CON. CON, control; CCM8, 8-week cirrhotic cardiomyopathy; LVESD, left ventricular end-systolic dimension; LVEDD, left ventricular end-diastolic dimension; IVS, interventricular septal dimension; LVPW, left ventricular posterior wall dimension; LAD, left atrial diameter; EF, ejection fraction; CO, cardiac output; DT, deceleration time; VE/VA, the mitral valve peak early diastolic velocity and late diastolic velocity ratio.

A

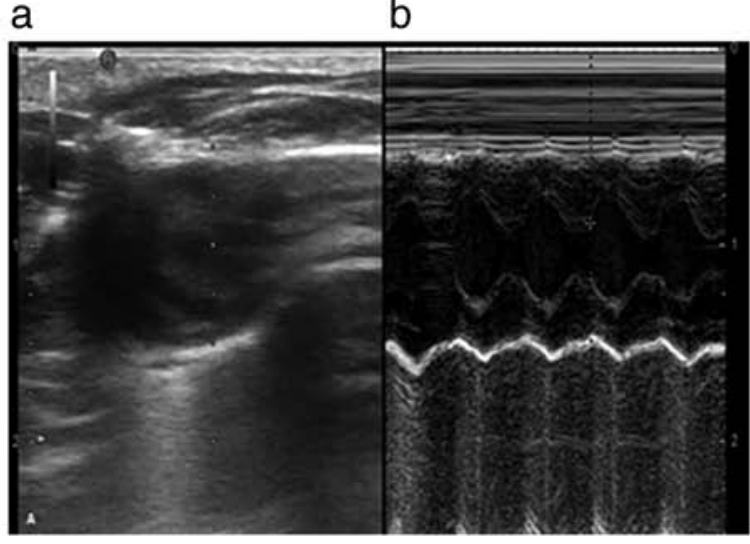

B

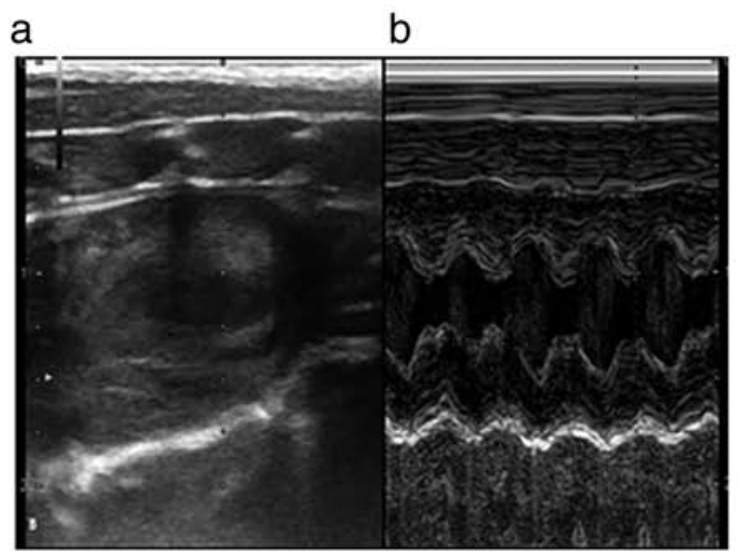

Figure 3. Representative ultrasound images measuring heart function in rats in the control and 8-week cirrhotic cardiomyopathy groups. (A) Control group, (B) 8-week cirrhotic cardiomyopathy group, (a) Two dimensional ultrasound and (b) M-mode ultrasound.

Patients with cirrhosis, especially those who had undergone liver transplantation previously received TIPS before, frequently have compromised circulatory systems and lead to fatal arrhythmia, heart failure, or even death (20). Therefore, studies into CCM warrants further study (29). Due to a combination of neurohumoral factors such as, troponin I, B-type natriuretic peptide (BNP) and pro-BNP, ventricular load is aggravated, which together with increased sodium retention and insufficient myocardial blood supply, result in myocardial hypertrophy and myocardial interstitial fibrosis (30). Ventricular remodeling is a pathological characteristic that occurs during CCM (31). Previous studies have shown that the proportion of heart enlargement in patients with cirrhosis is significantly increased compared with the healthy control group, where the structural cardiac changes are mainly focused in the left atrium and ventricle $(8,9)$. Extensive thickening of the left ventricular wall and histomorphological changes, including cardiac hypertrophy and interstitial/intracellular edema, can also be seen in patients with cirrhosis $(32,33)$. In the present study, the liver weight and heart weight of rats with cirrhosis were significantly lower compared with those in the control rats, but the cardiac coefficient (heart/body ratio) and left ventricular weight/heart weight ratio were significantly increased, suggesting the occurrence of myocardial remodeling in rats with cirrhosis to some extent. In addition, cirrhosis not only causes myocardial cell hypertrophy but can also cause myocardial interstitial hyperplasia (33). In the present study, rats with cirrhosis, myocardial collagen fibers were found to be disordered, which can lead to hyperplasia and stiffness (34). Therefore, myocardial compliance indicated by the enlargement of left atrium and the decrease of VE/VA ratio in the present study decreased, which caused diastolic dysfunction. In addition, an increase in the levels of collagen fibers may interfere with the arrangement, communication and connection of myocardial cells, adversely affecting the production and transmission of force to induce systolic dysfunction (34). Myocardial fibrosis is an important manifestation of ventricular remodeling and also an intrinsic cause of cardiac insufficiency that can lead to heart failure (35).

The present study also examined changes in conventional ultrasound parameters in rats 8 weeks after CCM induction. Among them, the LVESD, LVEDD, IVS, and LAD values were significantly increased whilst an increase in the levels of $\mathrm{CO}$ was observed. However, the ratio of VE/VA was decreased and the DT was prolonged. These results were consistent with results from the ultrasound diagnosis in patients with clinical cirrhosis in our previous study (7), suggesting that this modeling method can mimic myocardial damage in patients with cirrhosis and lead to cardiac function. In addition, this also suggests that high-frequency ultrasound can clearly detect the various indices of rat heart function. The hemodynamic indices such as CO, LVEF and VE/VA of rats with cirrhosis appeared 
Table IV. Changes in the expression of biomarkers for myocardial injury in rats with cirrhosis.

\begin{tabular}{lrrr}
\hline Biomarker & \multicolumn{1}{c}{ CON } & CCM4 & CCM8 \\
\hline Lactate dehydrogenase & $219.6 \pm 35.6$ & $407.1 \pm 40^{\mathrm{a}}$ & $567.4 \pm 47.3^{\mathrm{a}}$ \\
Creatine kinase-isoenzyme & $308.23 \pm 32.9$ & $451 \pm 66.2^{\mathrm{a}}$ & $658 \pm 50.5^{\mathrm{a}}$ \\
Cardiac tropinin T & $258.7 \pm 38.2$ & $398.5 \pm 56.3^{\mathrm{a}}$ & $603.3 \pm 48.4^{\mathrm{a}}$
\end{tabular}

${ }^{a} \mathrm{P}<0.01$ vs. CON. CON, control; CCM4, 4-week cirrhotic cardiomyopathy; CCM8, 8-week cirrhotic cardiomyopathy.
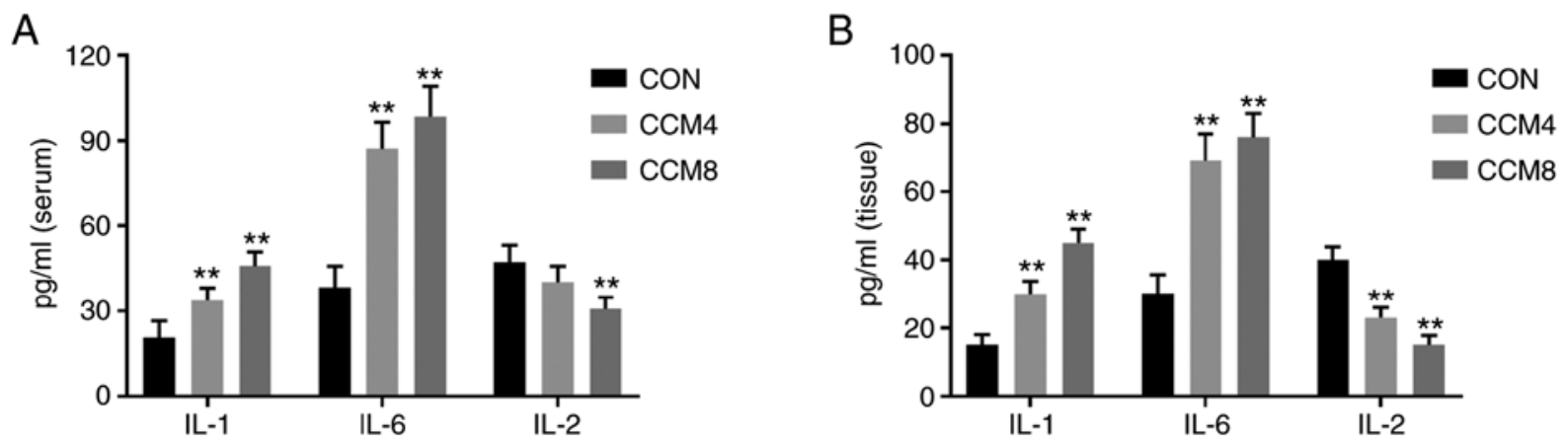

Figure 4. Changes in the biomarkers of myocardial injury in rats in the three treatment groups. ELISA was performed in (A) serum and (B) cardiac tissue samples. ${ }^{* *} \mathrm{P}<0.01$ vs. CON. CON, control; CCM4, 4-week cirrhotic cardiomyopathy; CCM8, 8-week cirrhotic cardiomyopathy.

A

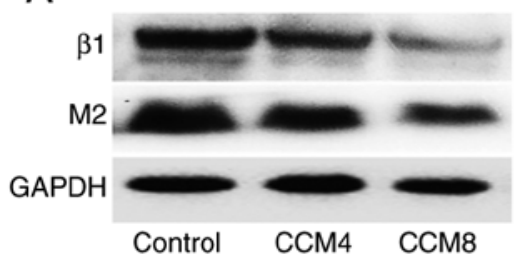

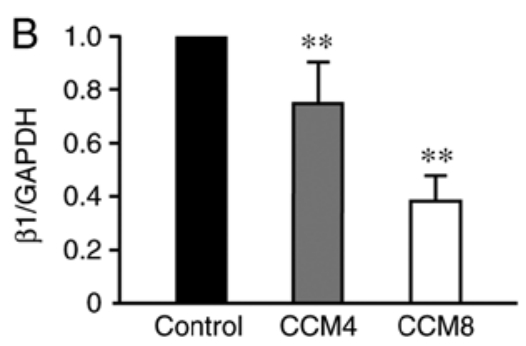

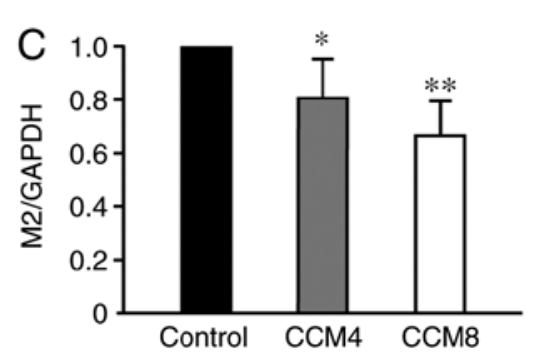

Figure 5. Expression levels of myocardial $\beta 1$ and $\mathrm{M} 2$ receptor proteins in rats in the three treatment groups. (A) Representative western blotting images. (B) $\beta 1$ and (C) M2 receptor expression was quantified. ${ }^{*} \mathrm{P}<0.05$ and ${ }^{* *} \mathrm{P}<0.01$ vs. CON. CON, control; CCM4, 4-week cirrhotic cardiomyopathy; CCM8, 8-week cirrhotic cardiomyopathy; $\beta 1, \beta 1$-adrenergic receptors; $\mathrm{M} 2$, muscarinic acetylcholine receptor.

to be lower than those of control rats, suggesting that this is an effective model for studying the pathogenesis of CCM.

Inflammatory responses are the body's major responses to tissue damage and serves an important role in cardiac damage (36). The degree of the inflammatory response is an important factor in determining the prognosis of patients with myocardial injuries (36). Proinflammatory factors IL-6 and IL- $1 \beta$ are not normally expressed in the myocardium, where their production and upregulation results in intrinsic stress and myocardial damage (37). Various reports (37-39) revealed that during cirrhosis, IL- 6 and IL- $1 \beta$ can be stimulated by endotoxemia and other factors such as lipopolysaccharide and $\mathrm{T}$ cell immunoglobulin and mucin domain 3, which can induce fibrosis in damaged myocardial fibers. The present study also revealed that the levels of IL- 6 and IL-1 $\beta$ in the serum and myocardial tissues of rats with cirrhosis were significantly higher compared with those in the CON group, which increased the severity of cirrhosis. By contrast, the anti-inflammatory factor IL-2 showed a significant decrease, suggesting that an aberrant inflammatory reaction is involved in the damage caused by cirrhosis to the myocardium and accelerated ventricular remodeling.

Cirrhosis causes hyperdynamic circulation and is regulated by neurohumoral factors (40). The hyperdynamic circulation begins in the portal venous bed as a consequence of portal hypertension due to the increased resistance to flow from altered hepatic vascular morphology of chronic liver disease (41). Previous studies have reported that myocardial $\beta$-adrenergic receptor $(\beta$-AR) downregulation may be an important pathogenic mechanism in cirrhosis-induced myocardial injury $(42,43)$. The adrenaline receptor subtype predominantly expressed in cardiac tissues is $\beta 1$, where its density reduction and/or desensitization is closely associated with the development of cardiovascular diseases, including heart failure and hypertension (44). The present study also found that the expression of the $\beta 1$-AR protein in the myocardium gradually decreased with prolongation of the CCM course. The heart is also dually regulated by the sympathetic and parasympathetic nervous systems, which antagonize each other to maintain the dynamic 
balance required for basal cardiac function under various stress-inducing states (45). In the present study, in addition to the reduction in the levels of $\beta 1-A R$ expression in CCM rat tissues, the expression of M2 receptor (M2), a major receptor for myocardial parasympathetic neurotransmitters (46), was also reduced. However, this downward trend was not as pronounced as that of $\beta 1$. These findings suggest that there is a decrease in autonomic regulation during cirrhosis-induced cardiac dysfunction, but the balance between sympathetic and parasympathetic in cardiac regulation requires further study.

To conclude, rat CCM model established by CCL4 treatment in the present study can simulate the pathogenesis of cirrhosis to cause myocardial damage. There was a clear sign of myocardial remodeling present in rats with CCM. High-frequency ultrasound electrocardiography can be used to effectively observe changes in cardiac functions in rats. The levels of pro-inflammatory factors IL-6 and IL-1 were increased, whilst the expression of $\beta 1-\mathrm{AR}$ protein and M2 receptor were decreased in rats with cirrhosis, suggesting that abnormal inflammatory reaction, autonomic regulation and other mechanisms may be involved in cirrhosis-related damage to the myocardium and accelerate ventricular remodeling.

\section{Acknowledgements}

Not applicable.

\section{Funding}

The present study was sponsored by the National Natural Science Foundation of China (grant no. 82001843).

\section{Availability of data and materials}

The datasets used and/or analyzed during the current study are available from the corresponding author on reasonable request.

\section{Authors' contributions}

SY and LS performed the experiments, analysis and interpretation of the data and revision for intellectual content. HW and $\mathrm{JJ}$ were involved in the experiments, and acquisition, analysis and interpretation of the data. QZ was responsible for the conception, design, and acquisition, analysis and interpretation of the data. SY and LS confirmed the authenticity of all the raw data. All authors read and approved the final manuscript.

\section{Ethics approval and consent to participate}

The present study was performed in strict accordance with the recommendations in the Guide for the Care and Use of Laboratory Animals of the National Institutes of Health. The animal use protocol has been reviewed and approved by the Institutional Animal Care and Use Committee of Xi'an Jiaotong University (Xi'an, China).

\section{Patient consent for publication}

Not applicable.

\section{Competing interests}

The authors declare that they have no competing interests.

\section{References}

1. Romanelli RG and Stasi C: Recent advancements in diagnosis and therapy of liver cirrhosis. Curr Drug Targets 17: 1804-1817, 2016.

2. Tsochatzis EA, Bosch $\mathrm{J}$ and Burroughs AK: Liver cirrhosis. Lancet 383: 1749-1761, 2014.

3. Kowalski HJ and Abelmann WH: The cardiac output at rest in Laennec's cirrhosis. J Clin Invest 32: 1025-1033, 1953.

4. Ruiz-del-Arbol L and Serradilla R: Cirrhotic cardiomyopathy. World J Gastroenterol 21: 11502-11521, 2015.

5. Khare J, Srivastava P, Wadhwa J and Deb P: Cardiac cirrhosis-an uncommon manifestation of common disease. OGH Reports 6: 28-30, 2017.

6. Jarkovska D, Bludovska M, Mistrova E, Krizkova V, Kotyzova D, Kubikova T, Slavikova J, Erek SN, Djordjevic A and Chottova Dvorakova M: Expression of classical mediators in hearts of rats with hepatic dysfunction. Can J Physiol Pharmacol 95: 1351-1359, 2017.

7. Li X, Yu S, Li L, Han D, Dai S and Gao Y: Cirrhosis-related changes in left ventricular function and correlation with the model for end-stage liver disease score. Int J Clin Exp Med 7: 5751-5757, 2014.

8. Cesari M, Fasolato S, Rosi S and Angeli P: Cardiac dysfunction in patients with cirrhosis: Is the systolic component its main feature? Eur J Gastroenterol Hepatol 27: 660-666, 2015.

9. Sampaio F, Pimenta J, Bettencourt N, Fontes-Carvalho R, Silva AP, Valente J, Bettencourt P, Fraga J and Gama V: Systolic and diastolic dysfunction in cirrhosis: A tissue-Doppler and speckle tracking echocardiography study. Liver Int 33: 1158-1165, 2013.

10. Gregolin CS, do Nascimento M, Borges de Souza SL, Ferreira Mota GA, Bomfim GF, de Azevedo Melo Luvizotto R, Sugizaki MM, Zanati Bazan SG, Salomé de Campos DH, Dias MC, et al: Myocardial dysfunction in cirrhotic cardiomyopathy is associated with alterations of phospholamban phosphorylation and IL-6 levels. Arch Med Res 52: 284-293, 2021.

11. Silvestre OM, Farias AQ, Ramos DS, Furtado MS, Rodrigues AC, Ximenes RO, de Campos Mazo DF, Yoshimura Zitelli PM, Diniz MA, Andrade JL, et al: $\beta$-blocker therapy for cirrhotic cardiomyopathy: A randomized-controlled trial. Eur J Gastroenterol Hepatol 30: 930-937, 2018.

12. Accornero F, van Berlo JH, Correll RN, Elrod JW, Sargent MA, York A, Rabinowitz JE, Leask A and Molkentin JD: Genetic analysis of connective tissue growth factor as an effector of transforming growth factor $\beta$ signaling and cardiac remodeling. Mol Cell Biol 35: 2154-2164, 2015.

13. Xiao H, Li H, Wang JJ, Zhang JS, Shen J, An XB, Zhang CC, Wu JM, Song Y, Wang XY, et al: IL-18 cleavage triggers cardiac inflammation and fibrosis upon $\beta$-adrenergic insult. Eur Heart J 39: 60-69, 2018.

14. Ziemssen T and Siepmann T: The investigation of the cardiovascular and sudomotor autonomis nervous system-a review. Front Neurol 10: 53, 2019.

15. Yang $\mathrm{CH}$, Ting WJ, Day $\mathrm{CH}$, Ju DT, Yeh YL, Chung LC, Tsai FJ, Tsai $\mathrm{CH}$, Tsai Y and Huang CY: SHSST cyclodextrin complex prevents the fibrosis effect on $\mathrm{CCl}_{4}$-induced cirrhotic cardiomyopathy in rats through TGF- $\beta$ pathway inhibition effects. Int J Mol Sci 15: 8037-8048, 2014.

16. Clark JD, Gebhart GF, Gonder JC, Keeling ME and Kohn DF: The 1996 guide for the care and use of laboratory animals. ILAR J 38: 41-48, 1997.

17. Kasarala G and Tillmann H: Standard liver tests. Clin Liver Dis (Hoboken) 8: 13-18, 2016.

18. Lo RC and Kim H: Histopathological evaluation of liver fibrosis and cirrhosis regression. Clin Mol Hepatal 23: 302-307, 2017.

19. Moller S and Henriksen JH: Cirrhotic cardiomyopathy. J Hepatol 53: 179-190, 2010.

20. Naqvi IH, Mahmood K, Naeem M, Vashwani AS and Ziaullah S: The heart matters when the liver shatters! Cirrhotic cardiomyopathy: Frequency, comparison, and correlation with severity of disease. Prz Gastroenterology 11: 247-256, 2016.

21. Berzigotti A: Advances and challenges in cirrhosis and portal hypertension. BMC Med 15: 200, 2017. 
22. Chayanupatkul $M$ and Liangpunsakul S: Cirrhotic cardiomyopathy: Review of pathophysiology and treatment. Hepatol Int 8: 308-315, 2014.

23. Wang Y, Hu Y, Zeng Z, Li Y, Su H, Li Y, Wang R, Zhang M, Yang $\mathrm{Y}$ and Deng J: Influence of androgen on myocardial apoptosis and expression of myocardial IR and IRS1 in chronic heart failure rat models. Mol Med Rep 17: 1057-1064, 2018.

24. Gassanov N, Caglayan E, Semmo N, Massenkeil G and Er F: Cirrhotic cardiomyopathy: A cardiologist's perspective. World J Gastroenterol 20: 15492-15498, 2014.

25. Xiao J, Wang F, Wong NK, He J, Zhang R, Sun R, Xu Y, Liu Y, Li W, Koike K, et al: Global liver disease burdens and research trends: Analysis from a Chinese perspective. J Hepatol 71 212-221, 2019

26. Wu L, Ong S, Talor MV, Barin JG, Baldeviano GC, Kass DA, Bedja D, Zhang H, Sheikh A, Margolick JB, et al: Cardiac fibroblasts mediate IL-17A-driven inflammatory dilated cardiomyopathy. J Exp Med 211: 1449-1464, 2014

27. Fede G, Privitera G, Tomaselli T, Spadaro L and Purrello F: Cardiovascular dysfunction in patients with liver cirrhosis. Ann Gastroenterol 28: 31-40, 2015.

28. Karagiannakis DS, Papatheodoridis G and Vlachogiannakos J: Recent advances in cirrhotic cardiomyopathy. Dig Dis Sci 60: $1141-1151,2015$

29. Liu H, Jayakumar S, Traboulsi M and Lee SS: Cirrhotic cardiomyopathy: Implications for liver transplantation. Liver Transpl 23: 826-835, 2017.

30. Saner FH, Neumann T, Canbay A, Trechmann JW, Hartmann M, Goerlinger K, Bertram S, Beckebaum S, Cicinnati V and Paul A: High brain-natriuretic peptide level predicts cirrhotic cardiomyopathy in liver transplant patients. Transpl Int 24 425-432, 2011

31. Yang YY and Lin HC: The heart: Pathophysiology and clinical implications of cirrhotic cardiomyopathy. J Chin Med Assoc 75: 619-623, 2012

32. Zaky A and Lang JD: Cirrhosis-associated cardiomyopathy. J Anesth Clin Res 3: 1-7, 2012.

33. Ortiz-Olvera NX, Castellanos-Pallares G, Gómez-Jiménez LM Cabrera-Muñoz ML, Méndez-Navarro J, Morán-Villota S and Dehesa-Violante M: Anatomical cardiac alterations in liver cirrhosis: An autopsy study. Ann Hepatol 10: 321-326, 2011.

34. Naveed M, Han L, Hasnat M, Baig MMFA, Wang W, Mikrani R, Zhiwei L, Sembatya KR, Xie D and Zhou X: Suppression of TGP on myocardial remodeling by regulating the NF- $\kappa \mathrm{B}$ pathway. Biomed Pharmacother 108: 1460-1468, 2018.

35. Briasoulis A, Mallikethi-Reddy S, Palla M, Alesh I and Afonso L: Myocardial fibrosis on cardiac magnetic resonance and cardiac outcomes in hypertrophic cardiomyopathy: A meta-analysis. Heart 101: 1406-1411, 2015

36. Jou C, Shah R, Figueroa A and Patel JK: The role of inflammatory cytokines in cardiac arrest. J Intensive Care Med 35 219-224, 2018
37. Bageghni SA, Hemmings KE, Zava N, Denton CP, Porter KE, Ainscough JFX, Drinkhill MJ and Turner NA: Cardiac fibroblast-specific p38 $\alpha$ MAP kinase promotes cardiac hypertrophy via a putative paracrine interleukin- 6 signaling mechanism. FASEB J 32: 4941-4954, 2018

38. Soresi M, Giannitrapani L, D'Antona F, Florena AM, La Spada E, Terranova A, Cervello M, D'Alessandro N and Montalto G: Interleukin-6 and its soluble receptor in patients with liver cirrhosis and hepatocellular carcinoma. World J Gastroenterol 12: 2563-2568, 2006.

39. Liu X, Li C, Zhu J, Li W and Zhu Q: Dysregulation of FTX/miR-545 signaling pathway downregulates Tim-3 and is responsible for the abnormal activation of macrophage in cirrhosis. J Cell Biochem: Oct 10, 2018 (Epub ahead of print)

40. Pagourelias ED, Sotiriou P, Papadopoulos CE, Cholongitas E Giouleme $\mathrm{O}$ and Vassilikos V: Left ventricular myocardial mechanics in cirrhosis: A speckle tracking echocardiographic study. Echocardiography 33: 223-232, 2016.

41. Blendis L and Wong F: The hyperdynamic circulation in cirrhosis: An overview. Pharmacol Ther 89: 221-231, 2001.

42. Moller S, Hove JD, Dixen U and Bendtsen F: New insights into cirrhotic cardiomyopathy. Int J Cardiol 167: 1101-1108, 2013.

43. Muñoz-OrtegaMH,Llamas-RamírezRW,Romero-Delgadillo NI, Elías-Flores TG, Tavares-Rodríguez EJ, Campos-Esparza MR, Cervantes-García D, Muñoz-Fernández L, Gerardo-Rodríguez M and Ventura-Juárez J: Doxazasin treatment attenuates carbon tetrachloride-induced liver fibrosis in hamsters through a decrease in transforming growth factor $\beta$ secretion. Gut Liver 10 : 101-108, 2016.

44. Communal C, Singh M, Menon B, Xie Z, Colucci WS and Singh K: Betalintegrins expression in adult rat ventricular myocytes and its role in the regulation of beta-adrenergic receptorstimulated apoptosis. J Cell Biochem 89: 381-388, 2003.

45. Parvaneh S, Howe CL, Toosizadeh N, Honarvar B, Slepian MJ, Fain M, Mohler J and Najafi B: Regulation of cardiac autonomic nervous system control across frailty statuses: A systematic review. Gerontology 62: 3-15, 2016.

46. Ma G, Wang Y, Hou D, Liu J, Zhang J, Xu L, Wang H, Zhao W, Zhang $Y$ and Zhang L: Association of autoantibodies against the M2-muscarinic receptor with long-term outcomes in peripartum cardiomyopathy patients: A 5-year prospective study. J Cardiol 74: 251-257, 2019.

This work is licensed under a Creative Commons Attribution-NonCommercial-NoDerivatives 4.0 International (CC BY-NC-ND 4.0) License. 